\title{
UY Ursae Majoris: A W-subtype W UMa system with a small mass ratio
}

\author{
Y. Yang ${ }^{1,2,3}$, Q. Liu ${ }^{1,2,3}$, and K.-C. Leung ${ }^{4}$ \\ 1 Yunnan Observatory, Chinese Academy of Sciences, Kunming, Yunnan Province, PR China \\ 2 United Laboratory of Optical Astronomy, Chinese Academy of Sciences, PR China \\ 3 National Astronomical Observatories, Chinese Academy of Sciences, PR China \\ 4 Department of Physics and Astronomy, University of Nebraska, Lincoln, USA
}

Received 10 November 2000 / Accepted 31 January 2001

\begin{abstract}
We present light curves and photometric solutions of the contact binary UY UMa in this paper. The light curves appear to exhibit a typical O'Connell effect, with Maximum I being $0.034 \mathrm{mag}(V)$ and $0.030 \mathrm{mag}(B)$ brighter than Maximum II, respectively, and Maximum I shifting to phase 0.26 . The light curves are analyzed by means of the latest version of the WD program. The results show that UY UMa is a W-subtype contact binary with a small mass ratio $q=0.134$. The asymmetry of the light curves is explained by star spot models. From the collected data of 9 UMa contact systems with a smaller mass ratio than 0.20 , the nature of the secondaries is analyzed. The results show that the luminosities and densities of the secondaries could be controlled by the primaries and that the smaller the mass ratio, the stronger the control.
\end{abstract}

Key words. stars: binaries - starspots - stars: variables: general

\section{Introduction}

The variable UY UMa was discovered by Beljawsky (1933). In GCVS (Kukarkin et al. 1970) UY UMa is a W UMa-type contact binary with a period of 0.3760 and the magnitude of $m_{v}=12.7^{\mathrm{m}}-12.9^{\mathrm{m}}$. Photometric data of this system are quite rare. Recently, some photoelectric times of minimum light were published (Agerer et al. 1996-2000). No photoelectric light curve or radial-velocity curve has yet been published. As the orbital period can be considered a short one given and the lack of light curves and photometric solutions of the system, UY UMa was included in the observational program of short period variables observed with the $100-\mathrm{cm}$ reflecting telescope at the Yunnan Observatory.

\section{Observation}

The observation of UY UMa in the $B$ and $V$ bands was carried out on 3 nights in March, 1999, with the PI1024 TKB CCD photometric system attached to the $100-\mathrm{cm}$ reflecting telescope at the Yunnan Observatory in China. The effective field of view of the photometric system is 6.5 square arcmin at the Cassegrain focus and its $B V$ color system approximates the standard Johnson $B V$ photometric system (Yang \& Li 1999). The coordinates of

Send offprint requests to: Y. Yang, e-mail: bily@public.km.yn.cn the comparison star and the check star used are listed in Table 1. The comparison star and the check star are so close to the variable that they are in the same field of the observation, together with the program star. The photometric standard stars HD 23386, HD 23731, HD 23161 and HD 23156 were observed nightly in $B V$ bands during the observation to check the quality of the transformation coefficients of the photometric system to those of Johnson's standard $U B V$ system.

The integration time for each image was $100 \mathrm{~s}$. A total of 62 images in $V$ band and 60 images in $B$ band were obtained for 3 nights in March, 1999. The aperture photometry package of IRAF was used to reduce the images. The reduced results show that the difference between the magnitude of the check star and that of the comparison star is constant within a probable error of \pm 0.008 mag for $V$ color and \pm 0.009 mag for $B$ color. Extinction corrections were not made since the comparison star is very close to the variable.

Table 1. The coordinates of the variable, comparison and check star

\begin{tabular}{lcc}
\hline star & $\mathrm{RA}(1999.0)$ & Dec. $(1999.0)$ \\
\hline variable & $13^{\mathrm{h}} 44^{\mathrm{m}} 35^{\mathrm{s}}$ & $55^{\circ} 13^{\prime} 31^{\prime \prime}$ \\
comparison star & 134447 & 551122 \\
check star & 134431 & 551319 \\
\hline
\end{tabular}




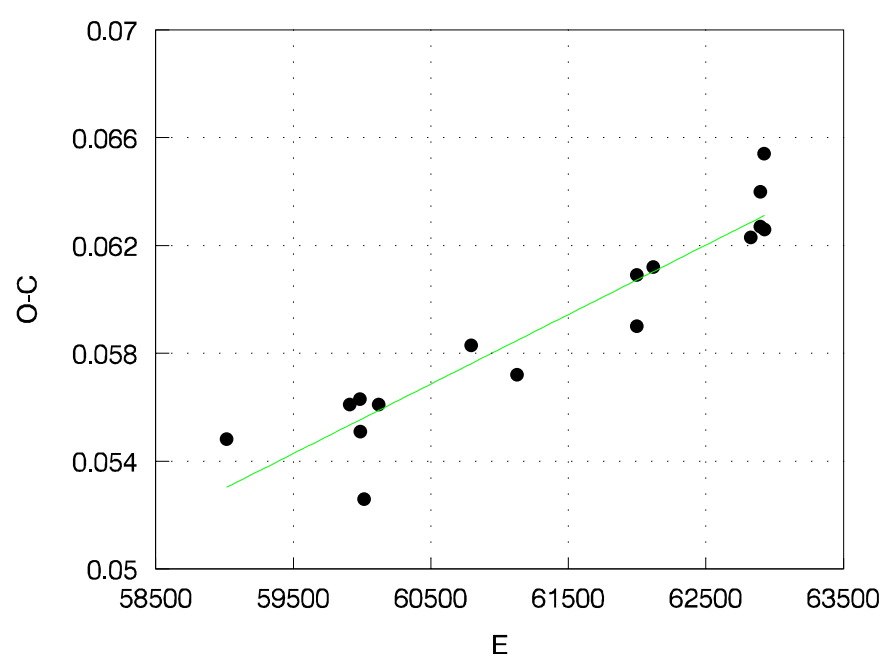

Fig. 1. The orbital period change of UY Uma

From the observations, two times of minimum light were derived by parabola fitting. The new times of the minimum light and the photoelectric times published by other authors are listed in Table 2 , in which the $(\mathrm{O}-\mathrm{C})_{1}$ values are calculated by means of the light element formula given in the GCVS (1993)

Min.I = HJD 2427586.4660+0.3760160 E.

Table 2. The times of minimum light of UY UMa

\begin{tabular}{lcccl}
\hline HJD 2400000+ & Min. & $(\mathrm{O}-\mathrm{C})_{1}$ & $(\mathrm{O}-\mathrm{C})_{2}$ & reference \\
\hline 49776.5410 & II & 0.0550 & 0.0017 & IBVS4383 \\
50113.6407 & I & 0.0561 & 0.0008 & IBVS4383 \\
50142.4061 & II & 0.0563 & 0.0008 & IBVS4472 \\
50142.5929 & I & 0.0551 & -0.0004 & IBVS4472 \\
50152.3668 & I & 0.0526 & -0.0030 & IBVS4472 \\
50192.4160 & II & 0.0561 & 0.0002 & IBVS4472 \\
50445.6650 & I & 0.0583 & 0.0007 & IBVS4562 \\
50570.5012 & I & 0.0572 & -0.0013 & IBVS4712 \\
50898.3909 & I & 0.0609 & 0.0002 & IBVS4712 \\
50898.5770 & II & 0.0590 & -0.0017 & IBVS4712 \\
50944.4531 & II & 0.0552 & 0.0002 & IBVS4712 \\
51209.5455 & II & 0.0623 & -0.0005 & IBVS4912 \\
51236.4310 & I & 0.0627 & -0.0004 & IBVS4912 \\
51236.6203 & II & 0.0640 & 0.0009 & IBVS4912 \\
51246.3962 & II & 0.0634 & 0.0003 & this paper \\
51247.3354 & I & 0.0626 & -0.0005 & this paper \\
\hline
\end{tabular}

From 14 photoelectric times of minimum light collected in the references and the new ones in the present article, the $(\mathrm{O}-\mathrm{C})_{1}$ values of the minima computed with the above ephemeris are plotted in Fig. 1. This diagram shows that the orbital period of UY UMa seems to be stable. A new ephemeris can be derived as follows:

Min.I = HJD 2451247.3356(11) + 0.37601846(22) E,

which is used to compute the phases of our observations and the $(\mathrm{O}-\mathrm{C})_{2}$ values in Table 2 .

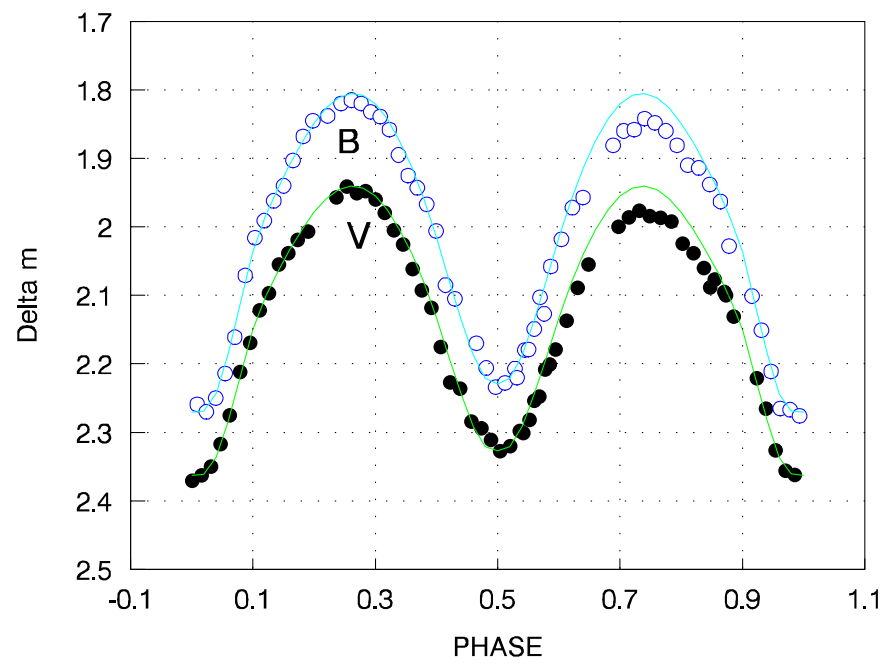

Fig. 2. The light curves of UY UMa. Solid circles: observational data; lines: model(see text for details)

A total of 62 yellow and 60 blue observations were obtained and listed in Tables 3 and 4 with their Heliocentric Julian Days, phases and magnitude differences between the variable and the comparison star. The light curve of the system is shown as the solid circle points in Fig. 2. The light curves appear to exhibit a typical O'Connell effect, with Maximum I being $0.034 \operatorname{mag}(V)$ and $0.030 \operatorname{mag}(B)$ brighter than Maximum II, respectively, and Maximum I shifting to phase 0.26 .

The usual standardization procedure of the observations of the UY UMa outside eclipse to the Johnson's standard $U B V$ system (Johnson 1963; Landolt 1973, 1983) is made to obtain the $B-V$ color of its outside eclipse. The transformation coefficients are determined by the observations of the standard stars (Yang \& Li 1999). In order to check the quality of the transformation coefficients, the standardization procedure of the observations of the observed photometric standard stars HD 23386, HD 23731, HD 23161 and HD 23156 was made. The results show that the standardization procedure is applicable to Johnson's standard $U B V$ system with the transformation coefficients of the photometric system used. No significant systematic difference appeared between the two systems. The maximum deviation of the standardization procedure is $\pm 0.012 \mathrm{mag}$ for $V$ and $\pm 0.018 \mathrm{mag}$ for $B$. The determined $B-V$ color of UY UMa is +0.608 at a phase of 0.25 and +0.617 at a phase of 0.75 .

\section{Photometric solutions}

Photometric solutions were obtained by means of the Wilson-Devinney program which includes a new reflection treatment, the option of using non-linear limb-darkening and the ability to adjust spot parameters. All the observations were used to compute the solutions. The convergence of the minimization procedure is obtained by means of the method of multiple subsets (Wilson \& Biermann 1976). 
Table 3. The CCD observations in $V$ band for UY UMa

\begin{tabular}{rrrrrrrrr}
\hline JD.(HEL) & PHASE & $\Delta m$ & $\begin{array}{c}\text { JD.(HEL) } \\
2451240+\end{array}$ & PHASE & $\Delta m$ & JD.(HEL) & PHASE & $\Delta m$ \\
$2451240+$ & & & & $2451240+$ & & \\
\hline 8.2991 & .2364 & 1.957 & 8.4238 & .5681 & 2.248 & 9.4005 & .1731 & 2.019 \\
.3054 & .2532 & 1.941 & .4304 & .5856 & 2.201 & .4069 & .1902 & 2.007 \\
.3115 & .2694 & 1.951 & 9.2781 & .8476 & 2.088 & 10.2912 & .5418 & 2.301 \\
.3172 & .2846 & 1.948 & .2866 & .8702 & 2.095 & .2980 & .5599 & 2.254 \\
.3231 & .3003 & 1.960 & .2925 & .8859 & 2.131 & .3049 & .5782 & 2.208 \\
.3288 & .3154 & 1.979 & .3065 & .9231 & 2.221 & .3113 & .5952 & 2.179 \\
.3345 & .3306 & 2.005 & .3123 & .9386 & 2.265 & .3178 & .6125 & 2.137 \\
.3402 & .3457 & 2.026 & .3183 & .9545 & 2.326 & .3247 & .6309 & 2.089 \\
.3460 & .3612 & 2.062 & .3241 & .9699 & 2.356 & .3312 & .6482 & 2.055 \\
.3517 & .3763 & 2.093 & .3299 & .9854 & 2.362 & .3497 & .6974 & 2.000 \\
& & & & & & & & \\
.3574 & .3915 & 2.118 & .3359 & .0013 & 2.370 & .3562 & .7146 & 1.986 \\
.3633 & .4072 & 2.157 & .3416 & .0165 & 2.363 & .3626 & .7317 & 1.976 \\
.3689 & .4221 & 2.227 & .3472 & .0314 & 2.350 & .3690 & .7487 & 1.985 \\
.3750 & .4383 & 2.236 & .3530 & .0468 & 2.317 & .3755 & .7660 & 1.987 \\
.3821 & .4572 & 2.284 & .3589 & .0625 & 2.275 & .3824 & .7843 & 1.992 \\
.3882 & .4734 & 2.294 & .3652 & .0793 & 2.212 & .3890 & .8019 & 2.024 \\
.3940 & .4888 & 2.311 & .3712 & .0952 & 2.169 & .3957 & .8197 & 2.039 \\
.3998 & .5042 & 2.328 & .3772 & .1112 & 2.122 & .4024 & .8375 & 2.060 \\
.4058 & .5202 & 2.320 & .3830 & .1266 & 2.097 & .4091 & .8553 & 2.077 \\
.4118 & .5361 & 2.298 & .3889 & .1423 & 2.055 & 10.4158 & .8731 & 2.100 \\
& & & & & & & & \\
8.4179 & .5524 & 2.282 & 9.3948 & .1580 & 2.093 & & & \\
\hline & & & & & & & &
\end{tabular}

Table 4. The CCD observations in $B$ band for UY UMa

\begin{tabular}{rrrrrrrrr}
\hline JD.(HEL) & PHASE & $\Delta m$ & $\begin{array}{c}\text { JD.(HEL) } \\
2451240+\end{array}$ & \multicolumn{7}{c}{ PHASE } & $\Delta m$ & JD.(HEL) & PHASE & $\Delta m$ \\
\hline 8.2938 & .2223 & 1.838 & 8.4207 & .5598 & 2.149 & 9.4039 & .1822 & 1.868 \\
.3020 & .2441 & 1.820 & .4268 & .5760 & 2.127 & 9.4098 & .1979 & 1.845 \\
.3083 & .2609 & 1.815 & 9.2895 & .8779 & 2.028 & 10.2873 & .5314 & 2.220 \\
.3144 & .2771 & 1.820 & .3036 & .9154 & 2.101 & .2945 & .5506 & 2.179 \\
.3202 & .2925 & 1.832 & .3094 & .9309 & 2.151 & .3016 & .5694 & 2.103 \\
.3260 & .3080 & 1.839 & .3154 & .9468 & 2.211 & .3081 & .5867 & 2.058 \\
.3317 & .3231 & 1.858 & .3211 & .9620 & 2.265 & .3146 & .6040 & 2.018 \\
.3373 & .3380 & 1.895 & .3271 & .9779 & 2.267 & .3214 & .6221 & 1.972 \\
.3432 & .3537 & 1.925 & .3328 & .9931 & 2.276 & .3280 & .6396 & 1.957 \\
.3488 & .3686 & 1.943 & .3388 & .0090 & 2.259 & .3464 & .6886 & 1.881 \\
& & & & & & & & \\
.3546 & .3840 & 1.967 & .3444 & .0239 & 2.270 & .3530 & .7061 & 1.860 \\
.3603 & .3992 & 2.056 & .3502 & .0394 & 2.250 & .3594 & .7232 & 1.858 \\
.3661 & .4146 & 2.085 & .3559 & .0545 & 2.214 & .3658 & .7402 & 1.842 \\
.3720 & .4303 & 2.155 & .3619 & .0705 & 2.161 & .3722 & .7572 & 1.848 \\
.3852 & .4654 & 2.170 & .3683 & .0875 & 2.071 & .3788 & .7747 & 1.860 \\
.3911 & .4811 & 2.206 & .3742 & .1032 & 2.016 & .3857 & .7931 & 1.881 \\
.3970 & .4968 & 2.234 & .3801 & .1189 & 1.991 & .3923 & .8106 & 1.910 \\
.4027 & .5119 & 2.228 & .3859 & .1343 & 1.962 & .3990 & .8285 & 1.914 \\
.4088 & .5282 & 2.207 & .3918 & .1500 & 1.940 & .4058 & .8466 & 1.938 \\
8.4148 & .5444 & 2.180 & 9.3976 & .1654 & 1.903 & .4124 & .8641 & 1.963 \\
\hline
\end{tabular}

The parameters adopted in the solutions are as follows: a temperature of $5900 \mathrm{~K}$ for Star 1 (the star eclipsed at Min.I), which corresponds to a $B-V$ color of 0.61 (Donald \& Thomas 1968), the values of the limb darkening coefficients $\left(x_{1}=x_{2}=0.640\right.$ for $V$ band and 0.780 for
$B$ band) (Claret et al. 1990), the values of the gravity darkening coefficients $\left(g_{1}=g_{2}=0.320\right)$ (Lucy 1967) and the values of the albedo $\left(A_{1}=A_{2}=0.500\right)$ (Rucinski $1969)$, corresponding to a $B-V$ color of 0.61 . The adjustable parameters are the orbital inclination, $\iota$, the mean 
temperature of Star $2, T_{2}$, the potential of the components, $\Omega_{1}$ and $\Omega_{2}$, and the monochromatic luminosity of Star 1 , $L_{1}$ (the Planck function is used to compute the luminosity).

Solutions are obtained for a series of fixed values of the mass ratio $q=m_{2} / m_{1}(0.10,0.20,0.30,0.40,0.50$, $0.60,0.70,0.80,0.90,0.10,1.20,1.40,1.60,2.00,2.40$, 2.60, 3.00, 3.40, 4.00, 5.00, 7.00, 9.00 and 10.00). Assuming that initially it was a detached system, the differential corrections begin from Mode 2, but the converged solutions were always obtained from the contact Mode 3 . The resulting sum $\Sigma$ of the square deviations of the converged solutions for each value of $q$ indicates that the fitting is best for $q=7.00$. As is well-known, the mass ratio and the inclination of a contact binary may compensate for each other. For UY UMa, the values of $\Sigma$ of a certain number for different combinations of $q$ and $i$ are shown in Table 5. At this point, the set of the adjustable parameters is expanded to include $q$. The mass ratio converges to a value of $q=7.4660$ in the final solution. The filling factor, $f=0.045$, has here the expression $\left(\Omega_{\text {in }}-\Omega\right) /\left(\Omega_{\text {in }}-\Omega_{\text {out }}\right)$ and varies from 0 to unity from the inner to the outer critical surface. This solution indicates that UY UMa is a W-subtype W UMa binary with a shallow contact degree. The photometric parameters are listed in the second column (unspotted) of Table 6 . The computed light curve using these parameters in the second column of the Table 6 is shown by solid lines in Fig. 2.

While the overall fit of the computed light curves is quite satisfactory, Fig. 2 shows obvious distortions in the observed light curves that seem to be due to the surface inhomogeneities of the components. Unequal quadrature light level, namely, the O'Connell effect, is known in many eclipsing binaries and several suggestions have been made to explain this effect by various authors. For UY UMa, the observed distortion, with Maximum II being fainter than Maximum I, may result from a cool or a hot region on either component. It is assumed that the spot is on star 1 or star 2 and we test several groups of dark spots or hot spots. We found four converged solutions with a cool spot on star 1 or star 2 and with a hot spot on star 1 or star 2, respectively. The solutions with the spots are listed in Table 6, where the solution labeled Dark 1 has a cool spot on the primary (more massive) star, labeled Dark 2 has a cool spot on the secondary star, the one labeled Hot 1 has a hot spot on the primary star and the one labeled Hot 2 has a hot spot on the secondary star. Also listed in Table 6 are spot labels and parameters (co-latitude, $\theta$, longitude, $\phi$, angular radius $\gamma$, all in degrees, and the temperature factor $T_{\mathrm{s}} / T_{*}$, with $T_{*}$ being the local effective temperature of the surrounding photosphere). The solution labeled Dark 1, i.e. the one with a cool spot on the primary (= more massive) star, turns out to be of slightly better quality than the others. The corresponding computed light curves are shown by the solid lines in Fig. 3. From Fig. 3 alone we can conclude that the O'Connell effect for UY UMa is due to a cool area appearing on the surface of the primary component.

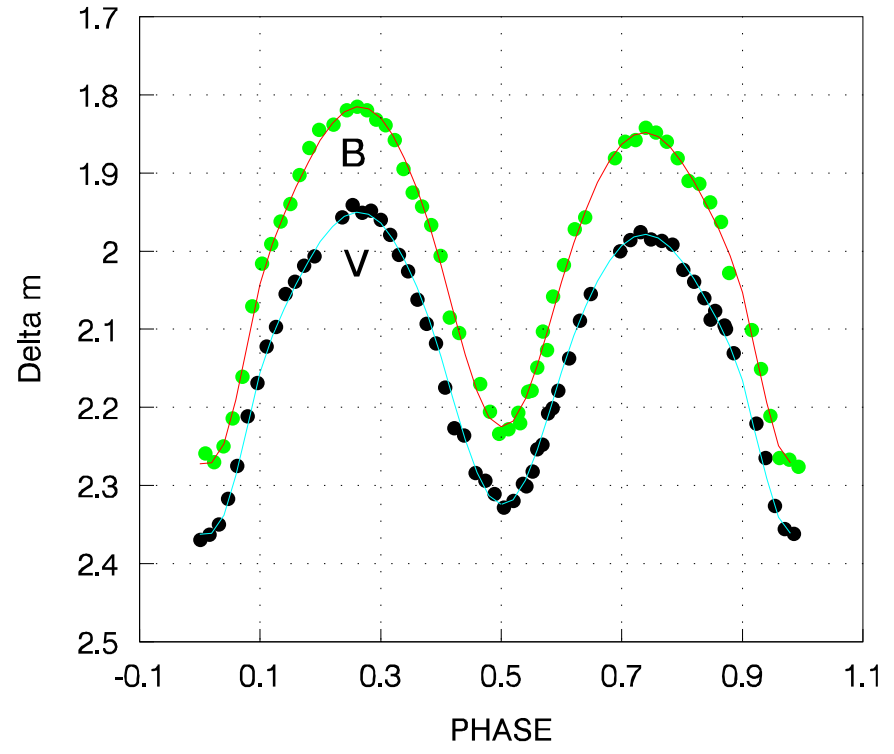

Fig. 3. As Fig. 2, but lines derived from the model with a dark spot on the primary component

\section{Discussion}

UY UMA is an important and interesting binary, mostly because of its W-subtype with a small mass ratio. In the present solution, the obtained mass ratio of UY UMA is 0.1339 for the unspotted solution, 0.1331 for the one labeled Dark 1, 0.1358 for the one labeled Dark 2, 0.1337 for the one labeled Hot 1 and 0.1334 for the one labeled Hot 2, respectively. These values are close to each other, therefore the mass ratio of 0.134 (average value) is adopted in the following calculation.

This is an interesting and important system to study the contact nature of systems with a small mass ratio. Since spectroscopic data for UY UMa are sparse, it is necessary to adopt the method introduced by Maceroni \& van't Veer (1996) to infer the physical parameters of the system. With their method, the total mass and total luminosity of the system can be derived and then from the photometric value of the mass ratio one can easily derive the physical parameter (such as the individual masses, the radii, etc.), which are listed in Table 7 .

Systems with a small mass ratio may be important for the study of the structure and evolution of a binary. The evolution of these systems is controlled by the primary, and the nature of the secondary is controlled by its contact with the primary. The small mass secondary has a large radius, resulting not from the evolution of reactions in the core of the secondary but rather from being heated by the radiation from the primary and from receiving energy through energy transfer from the primary to the secondary. Therefore, the structure and evolution of the secondaries of one of these binaries should be very interesting. Besides UY UMa, at least $8 \mathrm{~W}$ UMa type binaries with a mass ratio smaller than 0.2 (V677 Cen, FG Hya, XY Boo, AW UMa, TV Mus, RR Cen, MW Pav, and $\epsilon \mathrm{CrA}$ ) have been observed and analyzed. Some 
Table 5. The values of $\Sigma$ for different combinations of $q$ and $i$

\begin{tabular}{cccccccccccccc}
\hline$q$ & 0.20 & 0.40 & 0.60 & 0.80 & 1.00 & 1.40 & 2.00 & 2.40 & 3.00 & 4.00 & 5.00 & 7.00 & 9.00 \\
$i$ & 71.32 & 66.56 & 64.95 & 64.73 & 64.65 & 64.12 & 65.20 & 66.02 & 66.46 & 67.21 & 73.07 & 72.32 & \\
$\Sigma$ & 0.0170 & 0.0147 & 0.0137 & 0.0128 & 0.0122 & 0.0121 & 0.0114 & 0.0111 & 0.108 & 0.0106 & 0.0104 & 0.0098 & 0.0234 \\
\hline
\end{tabular}

Table 6. The photometric solutions of UY UMa

\begin{tabular}{lccccc}
\hline parameters & unspotted & Dark 1 & Dark 2 & Hot 1 & Hot 2 \\
\hline$q=m_{2} / m_{1}$ & $0.1339 \pm 0.0006$ & $0.1331 \pm 0.0006$ & $0.1358 \pm 0.0003$ & $0.1337 \pm 0.0006$ & $0.1334 \pm 0.0004$ \\
$i$ & $72.77 \pm 0.60$ & $73.38 \pm 0.41$ & $73.63 \pm 0.45$ & $72.14 \pm 0.42$ & $72.97 \pm 0.47$ \\
$T_{1}(\mathrm{~K})$ & $5500 \pm 25$ & $5486 \pm 12$ & $5508 \pm 13$ & $5514 \pm 16$ & $5519 \pm 13$ \\
$T_{2}(\mathrm{~K})$ & 5900 & 5900 & 5900 & 5900 & 5900 \\
$\Omega$ & $2.0543 \pm 0.0038$ & $2.0515 \pm 0.0022$ & $2.0593 \pm 0.0067$ & $2.0539 \pm 0.0059$ & $2.0535 \pm 0.0045$ \\
$f$ & 0.047 & 0.053 & 0.050 & 0.045 & 0.040 \\
$L_{1 V} /\left(L_{1 V}+L_{2 V}\right)$ & $0.7975 \pm 0.0018$ & $0.7928 \pm 0.0010$ & $0.7954 \pm 0.0011$ & $0.8006 \pm 0.0013$ & $0.8038 \pm 0.0 .0010$ \\
$L_{1 B} /\left(L_{1 B}+L_{2 B}\right)$ & $0.7859 \pm 0.0020$ & $0.7805 \pm 0.0011$ & $0.7839 \pm 0.0012$ & $0.7895 \pm 0.0014$ & $0.7931 \pm 0.0 .0012$ \\
$x_{1 V}=x_{2 V}$ & 0.640 & 0.640 & 0.640 & 0.640 & 0.640 \\
$x_{1 B}=x_{2 B}$ & 0.780 & 0.780 & 0.780 & 0.780 & 0.780 \\
$A_{1}=A_{2}$ & 0.500 & 0.500 & 0.500 & 0.500 & 0.500 \\
$g_{1}=g_{2}$ & 0.320 & 0.320 & 0.320 & 0.320 & 0.320 \\
$r_{1}$ (pole) & $0.5330 \pm 0.0001$ & $0.5357 \pm 0.0005$ & $0.5331 \pm 0.0004$ & $0.5325 \pm 0.0005$ & $0.5304 \pm 0.0005$ \\
$r_{1}$ (side) & $0.5960 \pm 0.0002$ & $0.5998 \pm 0.0008$ & $0.5963 \pm 0.0007$ & $0.5951 \pm 0.0008$ & $0.5918 \pm 0.0008$ \\
$r_{1}$ (back) & $0.6213 \pm 0.0003$ & $0.6263 \pm 0.0009$ & $0.6221 \pm 0.0008$ & $0.6202 \pm 0.0010$ & $0.6160 \pm 0.0009$ \\
$r_{2}$ (pole) & $0.2284 \pm 0.0002$ & $0.2310 \pm 0.0006$ & $0.2306 \pm 0.0005$ & $0.2275 \pm 0.0006$ & $0.2246 \pm 0.0006$ \\
$r_{2}$ (side) & $0.2408 \pm 0.0002$ & $0.2441 \pm 0.0007$ & $0.2434 \pm 0.0006$ & $0.2397 \pm 0.0008$ & $0.2362 \pm 0.0007$ \\
$r_{2}$ (back) & $0.3079 \pm 0.0008$ & $0.3219 \pm 0.0013$ & $0.3144 \pm 0.0012$ & $0.3044 \pm 0.0015$ & $0.2938 \pm 0.0013$ \\
$\Sigma$ & 0.008751 & 0.005056 & 0.005328 & 0.005258 & 0.005101 \\
$\phi$ & & $95.6 \pm 0.2$ & $56.8 \pm 0.7$ & $58.3 \pm 1.4$ & $93.4 \pm 0.8$ \\
$\theta$ & & $278.9 \pm 2.9$ & $102.4 \pm 0.6$ & $85.3 \pm 1.9$ & $272.7 \pm 1.2$ \\
$\gamma$ & & $13.3 \pm 0.3$ & $21.8 \pm 0.4$ & $11.1 \pm 0.3$ & $28.9 \pm 0.4$ \\
$T_{\mathrm{s}} / T_{*}$ & & $0.84 \pm 0.02$ & $0.82 \pm 0.03$ & $1.10 \pm 0.01$ & $1.10 \pm 0.01$ \\
\hline & & & & & \\
& & & & & \\
& & & & &
\end{tabular}

Table 7. The W UMa binaries with small mass ratio

\begin{tabular}{lccccccccccc}
\hline star & $p$ & $q$ & $M_{1}$ & $M_{2}$ & $R_{1}$ & $R_{2}$ & $L_{1}$ & $L_{2}$ & $D_{2}$ & $\alpha$ & reference \\
\hline V677 Cen & 0.3251 & 0.142 & 1.06 & 0.15 & 1.19 & 0.51 & 1.39 & 0.27 & 1.62 & 3.04 & $(1)$ \\
FG Hya & 0.3278 & 0.142 & 1.08 & 0.15 & 1.27 & 0.53 & 1.75 & 0.29 & 1.46 & 3.16 & $(2)(3)$ \\
UY UMa & 0.3760 & 0.134 & 1.19 & 0.16 & 1.40 & 0.63 & 1.58 & 0.42 & 0.91 & 3.54 & $(4)$ \\
TV Mus & 0.4457 & 0.150 & 1.32 & 0.20 & 1.66 & 0.75 & 3.14 & 0.69 & 0.67 & 3.44 & $(1)$ \\
XY Boo & 0.3705 & 0.182 & 1.49 & 0.27 & 1.47 & 0.63 & 5.17 & 1.01 & 1.30 & 2.33 & $(1)$ \\
AW UMa & 0.4387 & 0.072 & 1.52 & 0.11 & 1.60 & 0.53 & 6.06 & 0.56 & 1.05 & 4.20 & $(1)$ \\
$\epsilon$ CrA & 0.5914 & 0.112 & 1.76 & 0.20 & 2.20 & 0.79 & 11.07 & 1.08 & 0.57 & 3.62 & $(1)$ \\
RR Cen & 0.6057 & 0.180 & 1.80 & 0.32 & 2.15 & 0.96 & 11.44 & 2.19 & 0.53 & 2.86 & $(1)$ \\
MW Pav & 0.7950 & 0.182 & 2.13 & 0.39 & 2.70 & 1.31 & 22.10 & 5.05 & 0.24 & 3.25 & $(1)$ \\
\hline
\end{tabular}

(1) Maceroni \& Veer van’t (1996), (2) Yang \& Liu (2000), (3) Lu \& Rucinski (1999), (4) the present paper.

parameters of these systems are also listed in Table 7 , in which the density of the secondary component, $D_{2}$ in $\mathrm{g} / \mathrm{cm}^{3}$, is (Mochnacki 1981)

$D_{2}=\frac{0.019 q}{p^{2} r_{2}{ }^{3}(1+q)}$

Figures 4 and 5 show the observed luminosity and density of the secondaries as a function of the mass of the primaries (solid circles), in order to investigate the relation between the nature of the secondaries and the mass of the primaries. From Fig. 4, we see that the observed luminosity of the secondaries is related to the mass of the primaries (the solid curve in Fig. 4 is obtained by fitting the 4 exponent polynomial to the observed points). The very good fit between the theoretical curve and the observations suggests that the radiative luminosities from the secondaries is influenced by the primaries. Figure 5 shows 


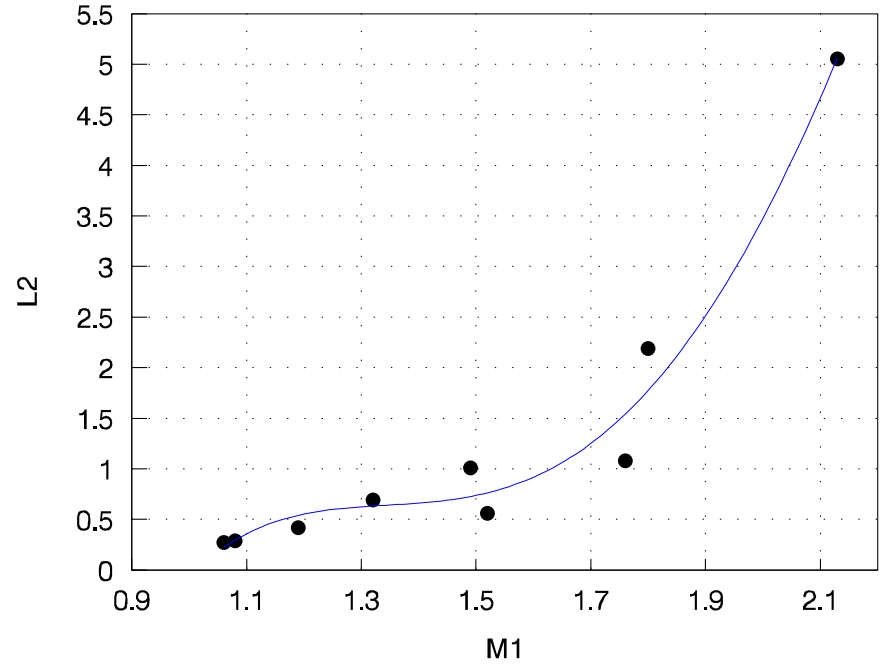

Fig. 4. The observed luminosity of the secondary as a function of the mass of the primary

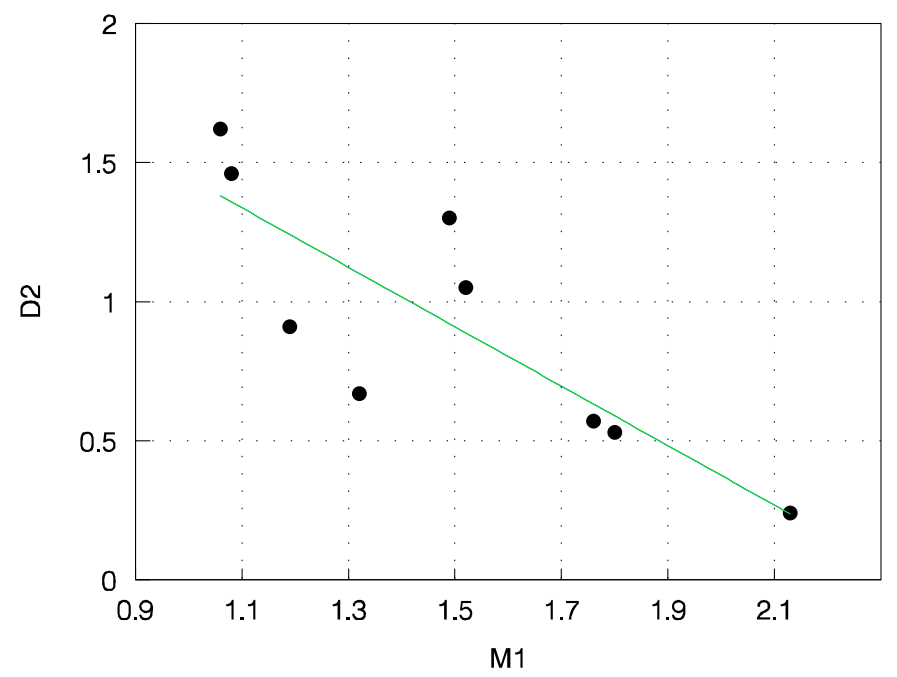

Fig. 5. The density of the secondary as a function of the mass of the primary

that the density $D_{2}$ of the secondary can be controlled by the primary, and the greater the mass of the primary, the smaller the density of the secondary, i.e., the nature of the secondary is strongly controlled by the primary.

Since the mass ratios of the present systems are different from each other (0.112 for $\epsilon \mathrm{CrA}$ and 0.182 for $\mathrm{XY}$ Boo), we investigated the variation of the secondaries with changes in mass ratio. A directed quality $\alpha$ is defined as $\alpha=R_{2} / R_{2}^{\circ}$, where $R_{2}$ is the observed radius of the secondary and $R_{2}{ }^{\circ}$ indicates the main-sequence radius for the same mass. According to Lacy $(1977), \mathrm{R}_{2}{ }^{\circ}=\mathrm{Cm}_{2}^{\beta}$, where $C=0.955$ and $\beta=0.917$ for $m_{2}<1.31_{\odot}$, and $C=1.025$ and $\beta=0.617$ for $m_{2}>1.31_{\odot} . \alpha$ is also listed in Table 7 . Similar to the density $D_{2}$, the quality $\alpha$ should also be controlled by the primaries. In Fig. $6, \alpha$ is shown as a function of the mass ratio. One can see that the greater the value of $\alpha$, the smaller the mass ratio. Thus, the

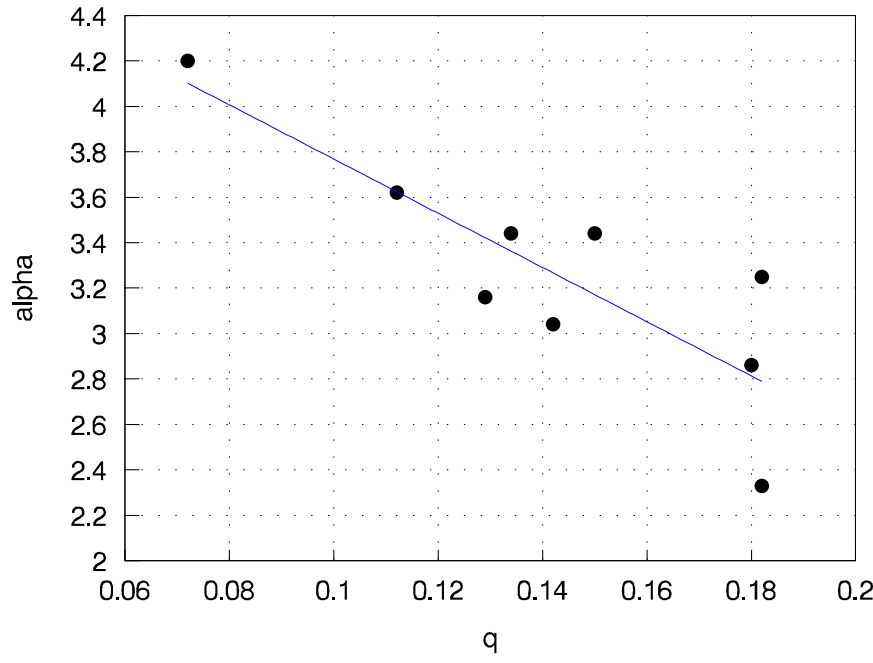

Fig. 6. The ratio of the observed radius to the main sequence radius for the same mass of the secondary as a function of the mass ratio

nature of the secondary is controlled by the primary for the systems with small mass ratios.

Our data show that UY UMa is a W-subtype contact binary with a small mass ratio. We present these photometric results for this system, but spectroscopic observations will be very necessary further to study its contact nature.

Acknowledgements. The authors would like to thank Dr. S. Qian and Dr. F. Li for their assistance with observations. The authors would also like to express their gratitude for the support from the Chinese National Science Foundation Committee and the Chinese Academy of Sciences.

\section{References}

Agerer, F., \& Hubscher, J. 1996, IBVS, No. 4383

Agerer, F., \& Hubscher, J. 1997, IBVS, No. 4472

Agerer, F., \& Hubscher, J. 1998, IBVS, No. 4562

Agerer, F., Dahm, M., \& Hubscher, J. 1999, IBVS, No. 4712

Agerer, F., \& Hubscher, J. 2000, IBVS, No. 4912

Donald, C. M., \& Thomas, F. A. 1968, ApJ, 151, 611

Beliawsky, S. 1933, Pi34, 196

Claret, A., \& Gimenez, A. 1990, A\&A, 230, 412

Johnson, H. L. 1963, In Basic Astronomical Data, ed. K. Aa. Strand (University of Chicago, Chicago), 204

Kukarkin, B. V., et al. 1970, The General Catalogue of Variable Stars, 3th ed. (Nauka, Moscow), vol. II, 410

Landolt, A. U. 1973, AJ, 78, 959

Landolt, A. U. 1983, AJ, 88, 439

Lu, W., \& Rucinski, S. M. 1999, AJ, 118, 515

Lucy, L. B. 1967, Zert. Astrophys., 65, 89

Maceroni, C., \& van't Veer, F. 1996, A\&A, 311, 523

Mochnacki, S. W. 1981, ApJ, 245, 650

Rucinski, S. M. 1969, A\&A, 19, 245

Wilson, R. E., \& Biermann 1976, A\&A, 48, 349

Yang, Y., \& Li, L. 1999, Publication of Yunnan Observatory, No. 1, 32

Yang, Y., \& Liu, Q. 2000, A\&AS, 144, 457 